Pacific

Journal of

Mathematics

\title{
ON BUCHSBAUM SIMPLICIAL AFFINE SEMIGROUPS
}

P.A. García-SÁnchez and J.C. Rosales 


\title{
ON BUCHSBAUM SIMPLICIAL AFFINE SEMIGROUPS
}

\author{
P.A. García-SÁnchez And J.C. Rosales
}

\begin{abstract}
We give an arithmetic characterization which allow us to determine algorithmically when the semigroup ring associated to a simplicial affine semigroup is Buchsbaum. This characterization is based on a test performed on the Apéry sets of the extremal rays of the semigroup. We use this method to obtain the cardinality of minimal presentations for semigroups with minimal Apéry set.
\end{abstract}

\section{Introduction.}

Let $S=\left\langle n_{1}, \ldots, n_{r}, n_{r+1}, \ldots, n_{r+m}\right\rangle \subseteq \mathbb{N}^{r}$ be a simplicial affine semigroup, that is $\mathrm{L}_{\mathbb{Q}_{0}^{+}}(S)=\mathrm{L}_{\mathbb{Q}_{0}^{+}}\left(\left\{n_{1}, \ldots, n_{r}\right\}\right)$, where $\mathrm{L}_{\mathbb{Q}_{0}^{+}}(A)=\left\{\sum q_{i} a_{i} \mid q_{i} \in\right.$ $\mathbb{Q}_{0}^{+}$and $\left.a_{i} \in A\right\}$. We assume that the elements $n_{1}, \ldots, n_{r}$ are linearly independent (otherwise $S$ can be embedded in $\mathbb{N}^{s}$ with $s<r$ ). This enables us to suppose that, up to isomorphism, $n_{i}=\alpha_{i} e_{i}$ with $\alpha_{i} \in \mathbb{N} \backslash\{0\}$ (as usual, $e_{i}$ denotes the element in $\mathbb{N}^{r}$ all of whose coordinates are equal to zero except the $i$-th which is equal to one). We will refer to $n_{1}, \ldots, n_{r}$ as the extremal rays of $S$.

Let $K[S]=\bigoplus_{s \in S} K y^{s}$ be the semigroup ring associated to $S$. We say that $S$ is Cohen-Macaulay if the ring $K[S]$ is Cohen-Macaulay. The same stands for the notions of Gorenstein, Buchsbaum and complete intersection semigroup. In [10] the authors gave a characterization of the Cohen-Macaulay and Gorenstein property for simplicial affine semigroups in terms of the Apéry sets of its extremal rays. In that paper we also studied the form and cardinality of a minimal system of generators of the defining ideals of this type of semigroup rings. The mentioned paper was inspired mostly in the characterization given by Goto, Suzuki and Watanabe in [5] and in the generalization given in [16] by Trung and Hoa. Here we focus our attention on Buchsbaum semigroups. There are a lot of papers devoted to the study of the structure of arithmetically Buchsbaum monomial curves (see for instance $[\mathbf{1}, \mathbf{7}, \mathbf{1 2}, \mathbf{1 5}]$ ). Using as a starting point the characterizations given by Trung in [14] and by Kamoi in [8], we present an alternative characterization of the Buchsbaum property in Theorem 5 (compare with Theorem 1.1, page 230, in [13]). This result is used later to achieve Theorem 9 which is the main result of this paper and presents an arithmetical characterization of the Buchsbaum property for simplicial affine semigroups 
in terms of the Apéry sets of their extremal rays. This main theorem provides us with a procedure for deciding whether or not a given simplicial affine semigroup is Buchsbaum. Finally these results are also used to give the exact cardinality of a minimal presentation of a Buchsbaum simplicial affine semigroup with minimal Apéry sets (using the notation in [4], these are Buchsbaum simplicial affine semigroups with maximal embedding dimension, and what we count here is the number of elements in a minimal system of generators of the defining ideal of the semigroup ring associated to the given monoid; see this reference for an explicit expression of the Hilbert polynomial for this semigroup ring). The number of elements of a minimal presentation for this kind of monoids is obtained from the Apéry sets of its extremal rays and in this way this result generalizes the bound given for Cohen-Macaulay simplicial affine semigroups with maximal codimension presented in $[\mathbf{1 0}]$.

\section{A characterization of Buchsbaum semigroups.}

For every $k \in \mathbb{N}$, define

$$
S_{k}=\left\{x \in \mathbb{N}^{r} \mid \text { there exists } 1 \leq i<j \leq r\right.
$$

$$
\text { such that } \left.x+k n_{i} \in S, x+k n_{j} \in S\right\} \text {. }
$$

The characterizations given here for Buchsbaum semigroups are based on the following result.

Proposition 1. The following conditions are equivalent.

(i) $S$ is a Buchsbaum semigroup.

(ii) $S_{2}+(S \backslash\{0\}) \subseteq S$.

(iii) For every $1 \leq i<j \leq r$ and $u, v \in S$, if $v+2 n_{j}=u+2 n_{i}$ then $v+(S \backslash\{0\}) \subseteq 2 n_{i}+S$

(iv) For every $x \in S$, if $x-2 n_{i}, x-2 n_{j} \in S$, for some $i \neq j \in\{1, \ldots, r\}$, then $x+n_{k}-\left(2 n_{i}+2 n_{j}\right) \in S$ for all $k \in\{1, \ldots, r+m\}$.

Proof. The equivalence between (i) and (ii) appears in Lemma 3 of [14]. The conditions (i) and (iii) are equivalent by Proposition 2.3 of [8]. Finally (iii) if and only if (iv) follows easily taking $x=v+2 n_{j}=u+2 n_{i}$.

There are several characterizations of the Cohen-Macaulay property similar to Proposition 1. Next we give one of them.

Proposition 2. The semigroup $S$ is a Cohen-Macaulay semigroup if and only if $S_{k}=S$ for every $k \in \mathbb{N} \backslash\{0\}$.

Proof. The fact that $S_{1}=S$ is equivalent to the Cohen-Macaulay property for $S$ is part of Corollary 4.4 in [16]. Besides, once $S_{1}=S$, one can prove that $S_{k}=S$ for all $k \geq 2$. 
In order to reformulate the Buchsbaum property for simplicial affine semigroups, we have to introduce some notation. The Apéry set of an element $n$ of $S$ is the set

$$
\mathrm{S}(n)=\{s \in S \mid s-n \notin S\} .
$$

The subgroup of $\mathbb{Z}^{r}$ generated by $\left\{n_{1}, \ldots, n_{r}\right\}$ is denoted by $\mathrm{G}\left(\left\{n_{1}, \ldots, n_{r}\right\}\right)$. Let $\bar{S}$ be the set of elements $x$ in $\mathbb{Z}^{r}$ such that $x+n_{i} \in S$ for all $i \in$ $\{1, \ldots, r+m\}$. Note that this set is a semigroup that contains $S$ and that

$$
\bar{S}=\left\{x \in \mathbb{N}^{r} \mid x+n_{i} \in S \text { for all } i \in\{1, \ldots, r+m\}\right\} .
$$

Since $\bar{S} \subseteq \mathbb{N}^{r}$ and $\mathrm{L}_{\mathbb{Q}_{0}^{+}}\left(n_{1}, \ldots, n_{r}\right)=\left(\mathbb{Q}_{0}^{+}\right)^{r}$, then $\bar{S}$ is simplicial as well.

As a consequence of the following result, $\bar{S}$ is finitely generated.

Lemma 3. Every submonoid $T$ of $\mathbb{N}^{r}$ containing $\left\{n_{1}, \ldots, n_{r}\right\}$ is finitely generated.

Proof. It is easy to see that since $T$ is simplicial, every element $t$ in $T$ can be written as $t=\sum_{i=1}^{r} a_{i} n_{i}+w$ for some $a_{i} \in \mathbb{N}$ and $w \in \bigcap_{i=1}^{r} \mathrm{~T}\left(n_{i}\right)$. If we want to demonstrate that $T$ is finitely generated, it suffices to prove that the set $\bigcap_{i=1}^{r} \mathrm{~T}\left(n_{i}\right)$ has a finite number of elements. For proving this, define in $\bigcap_{i=1}^{r} \mathrm{~T}\left(n_{i}\right)$ the following equivalence relation:

$$
x \sim y \text { if } x-y \in \mathrm{G}\left(\left\{n_{1}, \ldots, n_{r}\right\}\right) .
$$

Since there are at most $\alpha_{1} \cdots \alpha_{r}$ elements in $\mathbb{N}^{r}$ modulo $\mathrm{G}\left(\left\{n_{1}, \ldots, n_{r}\right\}\right)$, there is a finite number of $\sim$-classes in $\bigcap_{i=1}^{r} \mathrm{~T}\left(n_{i}\right)$. If we show that for every $x=\left(x_{1}, \ldots, x_{r}\right) \in \bigcap_{i=1}^{r} \mathrm{~T}\left(n_{i}\right)$, its $\sim$-class $[x]$ is finite, then we conclude the proof. Set $m=\left(x_{1} \bmod \alpha_{1}, \ldots, x_{r} \bmod \alpha_{r}\right)$. Clearly, for every element $y \in[x]$, there exists $a_{1}^{y}, \ldots, a_{r}^{y} \in \mathbb{N}$ such that $y=\sum_{i=1}^{r} a_{i}^{y} n_{i}+m$. If there exists $y, z \in[x]$ for which $\left(a_{1}^{y}, \ldots, a_{r}^{y}\right)<\left(a_{1}^{z}, \ldots, a_{r}^{z}\right)$, then $z=y+\sum_{i=1}^{r}\left(a_{i}^{z}-\right.$ $\left.a_{i}^{y}\right) n_{i} \notin \bigcap_{i=1}^{r} \mathrm{~T}\left(n_{i}\right)$, which contradicts $z \in \bigcap_{i=1}^{r} \mathrm{~T}\left(n_{i}\right)$. Hence the set of elements $A=\left\{\left(a_{1}^{y}, \ldots, a_{r}^{y}\right) \mid y \in[x]\right\}$ is a set of incomparable elements with respect to the usual partial order in $\mathbb{N}^{r}$ (product order). Using Dickson's lemma, it follows that there exists a finite number of elements in $A$ and thus a finite number of elements in $[x]$.

The following result indicates a connection between $S_{k}$ and $\bar{S}$.

\section{Lemma 4.}

$$
S \subseteq \bar{S} \subseteq S_{1} \subseteq(\bar{S})_{1} \subseteq S_{2} \subseteq(\bar{S})_{2} .
$$

Proof. Follows easily from the definitions of $S_{k}$ and $\bar{S}$.

The next statement justifies the definition of the semigroup $\bar{S}$.

Theorem 5. The semigroup $S$ is Buchsbaum if and only if $\bar{S}$ is CohenMacaulay. 
Proof. Necessity. By Proposition 1, we have $S_{2}+(S \backslash\{0\}) \subseteq S$, whence $S_{2} \subseteq \bar{S}$. Since the opposite inclusion always holds, $S_{2}=\bar{S}$ and by Lemma 4 , it follows that $\bar{S}=(\bar{S})_{1}$, which by Proposition 2 implies that $\bar{S}$ is CohenMacaulay.

Sufficiency. By Proposition $2, \bar{S}=(\bar{S})_{2}$. From Lemma 4 it follows that $S_{2}=\bar{S}$, which by the definition of $\bar{S}$ leads to $S_{2}+(S \backslash\{0\}) \subseteq S$. Proposition 1 asserts that $S$ is Buchsbaum.

From the characterization of the Cohen-Macaulay property, if $S$ is CohenMacaulay and $x+n_{i}, x+n_{j}$ are in $S$, for $i \neq j \in\{1, \ldots, r\}$, then is $x \in S$. Thus if $S$ is Cohen-Macaulay and $r \geq 2$, then $S=\bar{S}$. (The case $r=1$ is the numerical case and all numerical semigroups are Cohen-Macaulay and Buchsbaum.)

As an easy consequence of Theorem 5 we obtain the following remark (the proof is left to the reader).

Corollary 6. Let $T$ be a simplicial affine Cohen-Macaulay semigroup minimally generated by $\left\{n_{1}, \ldots, n_{r}, n_{r+1}, \ldots, n_{r+m}\right\}$ and $A$ be a nonempty subset of $\left\{n_{r+1}, \ldots, n_{r+m}\right\}$. Then $T \backslash A$ is Buchsbaum but not Cohen-Macaulay.

We illustrate this with an example.

Example 7. Let $S$ be the semigroup finitely generated by

$$
\{(2,0),(0,2),(1,1)\} \text {. }
$$

Clearly $S$ is Cohen-Macaulay (it is even a complete intersection). The semigroup $S \backslash\{(1,1)\}$ can be generated by

$$
\{(2,0),(0,2), 2(1,1), 3(1,1),(2,0)+(1,1),(0,2)+(1,1)\},
$$

whence $S \backslash\{(1,1)\}=\langle(2,0),(0,2),(3,1),(1,3)\rangle$ is Buchsbaum but not CohenMacaulay.

\section{How to determine whether a simplicial affine semigroup is Buchsbaum.}

As we did in the previous section, we define in $\bigcap_{i=1}^{r} \mathrm{~S}\left(n_{i}\right)$ the following binary relation:

$$
x \sim y \text { if } x-y \in \mathrm{G}\left(\left\{n_{1}, \ldots, n_{r}\right\}\right) .
$$

The next result is used in [10] for giving a procedure for determining whether a simplicial affine semigroup is Cohen-Macaulay.

Proposition 8. The following statements are equivalent.

(i) $S$ is a Cohen-Macaulay semigroup.

(ii) For any $s \in S$ and $i \neq j \in\{1, \ldots, r\}$, if $s-n_{i}$ and $s-n_{j}$ are in $S$ then $s-\left(n_{i}+n_{j}\right)$ also belongs to $S$. 
(iii) For every element $s \in S$ there exists a unique element $\left(a_{1}, \ldots, a_{r}\right) \in$ $\mathbb{N}^{r}$ and a unique element $w$ in $\bigcap_{i=1}^{r} \mathrm{~S}\left(n_{i}\right)$ such that $s=\sum_{i=1}^{r} a_{i} n_{i}+w$. (iv) For every $x, y \in \bigcap_{i=1}^{r} \mathrm{~S}\left(n_{i}\right)$, if $x-y \in \mathrm{G}\left(\left\{n_{1}, \ldots, n_{r}\right\}\right)$, then $x=y$.

(v) For every $x \in \bigcap_{i=1}^{r} \mathrm{~S}\left(n_{i}\right),[x]=\{x\}$.

Proof. The equivalence between (i)-(iv) appears in [10]. Condition (v) is a reformulation of (iv).

The next proposition shows what happens in the Buchsbaum case.

Theorem 9. The affine semigroup $S$ is Buchsbaum if and only if, for every $x \in \bigcap_{i=1}^{r} \mathrm{~S}\left(n_{i}\right)$, if $\#[x] \geq 2$, then there exists $m \in \bar{S}$ such that $[x]=$ $\left\{m+n_{1}, \ldots, m+n_{r}\right\}$.

Proof. Necessity. Let us assume that $\#[x] \geq 2$. By Theorem $5, \bar{S}$ is CohenMacaulay (recall that $\bar{S}$ is a simplicial affine semigroup whose extremal rays are the extremal rays of $S$ ). Proposition 8 ensures that there exist unique $m \in \bigcap_{i=1}^{r} \overline{\mathrm{S}}\left(n_{i}\right)$ and $\left(a_{1}, \ldots, a_{r}\right) \in \mathbb{N}^{r}$ such that $x=\sum_{i=1}^{r} a_{i} n_{i}+m$. In particular, this implies that $x-m \in \mathrm{G}\left(\left\{n_{1}, \ldots, n_{r}\right\}\right)$, whence $y-m \in$ $\mathrm{G}\left(\left\{n_{1}, \ldots, n_{r}\right\}\right)$ for all $y \in[x]$. We show that in this case $m$ cannot be in $S$. If this were not the case, then $a_{1}=\cdots=a_{r}=0$, since $x \in \bigcap_{i=1}^{r} \mathrm{~S}\left(n_{i}\right)$. Thus $m=x$. Recall that $\#[x] \geq 2$ and hence there exists $y \in[x] \backslash\{x\}$. Using once more Proposition 8, there exist $m^{\prime} \in \bigcap_{i=1}^{r} \overline{\mathrm{S}}\left(n_{i}\right)$ and $\left(b_{1}, \ldots, b_{r}\right) \in \mathbb{N}^{r}$ such that $y=\sum_{i=1}^{r} b_{i} n_{i}+m^{\prime}$. It follows that $m^{\prime}-m=(x-m)+(y-$ $x)+\left(m^{\prime}-y\right) \in \mathrm{G}\left(\left\{n_{1}, \ldots, n_{r}\right\}\right)$. Condition (v) of Proposition 8 ensures that $[m]=\{m\}$, which leads to $m=m^{\prime}$. Therefore $b_{1}=\cdots=b_{r}=0$, since $y \in[x] \subseteq \bigcap_{i=1}^{r} \mathrm{~S}\left(n_{i}\right)$. This means that $y=m=x$, which contradicts $y \neq x$.

We show next that $\left\{m+n_{1}, \ldots, m+n_{r}\right\} \subseteq[x]$. Since $m \in \bar{S}$, we get that $m+n_{k} \in S$ for all $1 \leq k \leq r$. The affine semigroup $S$ is simplicial and for this reason there exists $\left(c_{1}, \ldots, c_{r}\right) \in \mathbb{N}^{r}$ and $w \in \bigcap_{i=1}^{r} \mathrm{~S}\left(n_{i}\right)$ such that $m+n_{1}=\sum_{i=1}^{r} c_{i} n_{i}+w$ (observe that this forces $w$ to be in $[x]$ ). In addition, $w \in \bar{S}$ and $\bar{S}$ is Cohen-Macaulay, which by Proposition 8 implies that there exist $\left(d_{1}, \ldots, d_{r}\right) \in \mathbb{N}^{r}$ and $m^{\prime} \in \bigcap_{i=1}^{r} \overline{\mathrm{S}}\left(n_{i}\right)$ such that $w=\sum_{i=1}^{r} d_{i} n_{i}+m^{\prime}$. As before, we can deduce that $m=m^{\prime}$. It follows $m+n_{1}=\left(c_{1}+d_{1}\right) n_{1}+$ $\cdots+\left(c_{r}+d_{r}\right) n_{r}+m$. From Proposition 8 we get that $c_{1}+d_{1}=1$ and that $c_{2}+d_{2}=\cdots=c_{r}+d_{r}=0$. This leads to $w=m+n_{1} \in[x]$. Similarly it is shown that $m+n_{i} \in[x]$ for all $i \in\{2, \ldots, r\}$.

For the opposite inclusion, take $y \in[x]$. Then $y \in S \subseteq \bar{S}$. By the same argument used above, there exists $\left(a_{1}, \ldots, a_{r}\right) \in \mathbb{N}^{r}$ for which $y=$ $\sum_{i=1}^{r} a_{i} n_{i}+m$. The fact that $y \in S$ implies that $\sum_{i=1}^{r} a_{i} \geq 1$ and $y \in$ $\bigcap_{i=1}^{r} \mathrm{~S}\left(n_{i}\right)$ forces $\sum_{i=1}^{r} a_{i}=1$. Hence $y=m+n_{i}$ for some $i \in\{1, \ldots, r\}$.

Sufficiency. Define

$$
A=\left\{m_{[x]} \mid[x] \in \bigcap_{i=1}^{r} \mathrm{~S}\left(n_{i}\right) / \sim\right\}
$$




$$
\text { where } m_{[x]}= \begin{cases}x & \text { if } \#[\mathrm{x}]=1, \\ m & \text { if }[\mathrm{x}]=\left\{\mathrm{m}+\mathrm{n}_{1}, \ldots, \mathrm{m}+\mathrm{n}_{\mathrm{r}}\right\} .\end{cases}
$$

From the definition of $A$, any two of its elements are incongruent modulo $\mathrm{G}\left(\left\{n_{1}, \ldots, n_{r}\right\}\right)$. If we prove that $\bigcap_{i=1}^{r} \overline{\mathrm{S}}\left(n_{i}\right) \subseteq A$, we get that $\bigcap_{i=1}^{r} \overline{\mathrm{S}}\left(n_{i}\right)$ fulfills the same condition, which by Proposition 8 means that $\bar{S}$ is CohenMacaulay and by Theorem 5 that $S$ is Buchsbaum. Thus it suffices to show that $\bigcap_{i=1}^{r} \overline{\mathrm{S}}\left(n_{i}\right) \subseteq A$. Take $x \in \bigcap_{i=1}^{r} \overline{\mathrm{S}}\left(n_{i}\right)$. Then $x \in \bar{S}$, whence $x+n_{1}, x+n_{2} \in S$. Since $S$ is simplicial, there exist $\left(c_{1}, \ldots, c_{r}\right),\left(d_{1}, \ldots, d_{r}\right) \in$ $\mathbb{N}^{r}$ and $w, w^{\prime} \in \bigcap_{i=1}^{r} \mathrm{~S}\left(n_{i}\right)$ such that $x+n_{1}=\sum_{i=1}^{r} c_{i} n_{i}+w$ and $x+n_{2}=$ $\sum_{i=1}^{r} d_{i} n_{i}+w^{\prime}$. It follows that $w^{\prime} \in[w]$. By the definition of $A$, there exists $m \in A$ for which $w=m$ or $w=m+n_{i}$ for some $i \in\{1, \ldots, r\}$ and $m^{\prime} \in A$ such that $w^{\prime}=m^{\prime}$ or $w^{\prime}=m^{\prime}+n_{j}$ for some $j \in\{1, \ldots, r\}$. In any case, since $w^{\prime} \in[w]$, we have $m=m^{\prime}$. Thus both $x+n_{1}$ and $x+n_{2}$ can be written as $x+n_{1}=\sum_{i=1}^{r} a_{i} n_{i}+m$ and $x+n_{2}=\sum_{i=1}^{r} b_{i} n_{i}+m$ for some $\left(a_{1}, \ldots, a_{r}\right),\left(b_{1}, \ldots, b_{r}\right) \in \mathbb{N}^{r}$, which this leads to

$$
a_{1} n_{1}+\left(a_{2}+1\right) n_{2}+a_{3} n_{3}+\cdots+a_{r} n_{r}=\left(b_{1}+1\right) n_{1}+b_{2} n_{2}+\cdots+b_{r} n_{r} .
$$

Since $\left\{n_{1}, \ldots, n_{r}\right\}$ is a basis of $\mathbb{Q}^{r}$, we get that $a_{1}=b_{1}+1$, which implies that $a_{1} \geq 1$. Hence $x=\left(a_{1}-1\right) n_{1}+a_{2} n_{2}+\cdots+a_{r} n_{r}+m$. In addition, $m \in \bar{S}$ and $x \in \bigcap_{i=1}^{r} \overline{\mathrm{S}}\left(n_{i}\right)$, which forces $x$ to be equal to $m$.

If we know $\bigcap_{i=1}^{r} \mathrm{~S}\left(n_{i}\right)$, then we can check for every $x \in \bigcap_{i=1}^{r} \mathrm{~S}\left(n_{i}\right)$, whether $\#[x]=1$ or $[x]=\left\{m+n_{1}, \ldots, m+n_{r}\right\}$ for some $m \in \bar{S}$. If this is not the case, then $S$ is not Buchsbaum. In $[\mathbf{3}, \mathbf{1 0}]$ an algorithm for computing the set $\bigcap_{i=1}^{r} \mathrm{~S}\left(n_{i}\right)$ is presented. This idea is based on the fact that

$$
\bigcap_{i=1}^{r} \mathrm{~S}\left(n_{i}\right) \subseteq\left\{\sum_{i=1}^{m} \gamma_{r+i} n_{r+i} \mid \gamma_{r+i}<c_{r+i} \text { for all } i \in\{1, \ldots, m\}\right\},
$$

where $c_{r+i}=\min \left\{k \in \mathbb{N}-\{0\}: k n_{r+i} \in\left\langle n_{1}, n_{2}, \ldots, n_{r}\right\rangle\right\} \leq \alpha_{1} \cdots \alpha_{r}$.

Thus Theorem 9, together with the algorithm for computing $\bigcap_{i=1}^{r} \mathrm{~S}\left(n_{i}\right)$, constitutes a method for deciding whether a simplicial affine semigroup is Buchsbaum.

Example 10. Let $S=\langle(2,0),(0,1),(1,2),(3,1)\rangle$. We compute $\mathrm{S}((2,0)) \cap$ $\mathrm{S}((0,1))$ as explained in $[\mathbf{1 0}]$ and obtain

$$
\mathrm{S}((2,0)) \cap \mathrm{S}((0,1))=\{(0,0),(1,2),(3,1)\} .
$$

Observe that

$[(0,0)]=\{(0,0)\},[(1,2)]=\{(1,2),(3,1)\}=\{(1,1)+(2,0),(1,1)+(0,1)\}$.

Taking $m=(1,1), m+(2,0), m+(0,1), m+(1,2), m+(3,1) \in S$ and hence $m \in \bar{S}$. By Theorem $9, S$ is Buchsbaum but not Cohen-Macaulay, since $\#[(1,2)] \neq 1$ (Proposition 8). 
The condition $\#[x] \in\{1, r\}$ is not sufficient for $S$ to be Buchsbaum, as the following example shows.

Example 11. Let $S=\langle(2,0),(0,2),(3,1),(1,3),(1,2)\rangle$. Using the procedure presented in [10] to compute $\bigcap_{i=1}^{r} \mathrm{~S}\left(n_{i}\right)=\mathrm{S}((2,0)) \cap \mathrm{S}((0,2))$, we get

$$
\mathrm{S}((2,0)) \cap \mathrm{S}((0,2))=\{(0,0),(3,1),(1,3),(1,2),(4,3),(2,5)\} .
$$

It follows that

$$
\begin{aligned}
& {[(0,0)]=\{(0,0)\}, \quad[(3,1)]=\{(3,1),(1,3)\},} \\
& {[(1,2)]=\{(1,2)\}, \quad[(4,3)]=\{(4,3),(2,5)\} .}
\end{aligned}
$$

By looking at $[(3,1)]$, the only possible candidate to be $m$ is $(1,1)$. However, $m+(1,2)=(2,3) \notin S$, which by Theorem 9 implies that $S$ is not Buchsbaum, since $m \notin \bar{S}$.

\section{Buchsbaum semigroups with minimal Apéry set.}

In the sequel we assume that $\left\{n_{1}, \ldots, n_{r}, n_{r+1}, \ldots, n_{r+m}\right\}$ is a minimal system of generators of $S$. By the definition of $\bigcap_{i=1}^{r} \mathrm{~S}\left(n_{i}\right)$, this implies that $\left\{n_{r+1}, \ldots, n_{r+m}\right\}$ is included in $\bigcap_{i=1}^{r} \mathrm{~S}\left(n_{i}\right) \backslash\{0\}$. We say that $S$ has minimal Apéry set if

$$
\left\{0, n_{r+1}, \ldots, n_{r+m}\right\}=\bigcap_{i=1}^{r} \mathrm{~S}\left(n_{i}\right) .
$$

Here we transfer a result known for Cohen-Macaulay simplicial affine semigroups fulfilling this condition to the Buchsbaum case. To this end, we need to recall some basic concepts in order to fix notation.

Let $\varphi$ be the map defined by

$$
\varphi: \mathbb{N}^{r+m} \rightarrow S, \varphi\left(a_{1}, \ldots, a_{r+m}\right)=\sum_{i=1}^{r+m} a_{i} n_{i},
$$

and denote its kernel congruence by $\sigma$. Then $S$ is isomorphic to $\mathbb{N}^{r+m} / \sigma$.

We say that $\rho$ is a minimal system of generators of $\sigma$ if $\rho$ generates $\sigma$ and its cardinal is minimal among the cardinal of the sets generating $\sigma$. In this case we also say that $\rho$ is a minimal presentation of $S$. It can be shown that $\# \rho \geq r+m-r=m$ (see [6]).

Let $n \in S-\{0\}$. Define the graph $G_{n}$ as the graph whose vertices are

$$
\mathrm{V}\left(G_{n}\right)=\left\{n_{i} \mid n-n_{i} \in S, i \in\{1, \ldots, r+m\}\right\}
$$

and whose edges are

$$
\mathrm{E}\left(G_{n}\right)=\left\{\overline{n_{i} n_{j}} \mid n-\left(n_{i}+n_{j}\right) \in S, i, j \in\{1, \ldots, r+m\}, i \neq j\right\} .
$$

Define $\rho_{n}$ as follows.

1) If $G_{n}$ is connected, then $\rho_{n}=\emptyset$. 
2) If $G_{n}$ is not connected and $G_{n}^{1}, \ldots, G_{n}^{t}$ are the connected components of $G_{n}$, then choose a vertex $n_{j_{i}} \in \mathrm{V}\left(G_{n}^{i}\right)$ and an element $\alpha_{i}^{n}=$ $\left(a_{1}^{i}, \ldots, a_{r+m}^{i}\right) \in \mathbb{N}^{r+m}$ such that $\varphi\left(\alpha_{i}^{n}\right)=n$ and $a_{j_{i}}^{i} \neq 0$; define

$$
\rho_{n}=\left\{\left(\alpha_{2}^{n}, \alpha_{1}^{n}\right), \ldots,\left(\alpha_{t}^{n}, \alpha_{1}^{n}\right)\right\} .
$$

Take $\rho=\bigcup_{n \in S} \rho_{n}$. Then $\rho$ is a minimal system of generators of $\sigma$ (this follows from a straightforward generalization presented in $[\mathbf{3}, \mathbf{1 1}]$ of the results given in [9]). Furthermore, every minimal system of generators of $\sigma$ has the same cardinality.

Example 12. Let

$$
S=\langle(2,0),(0,1),(1,2),(3,1)\rangle \subseteq \mathbb{N}^{2} .
$$

The elements $n \in S$ for which $G_{n}$ is not connected are $(3,2),(6,2),(4,3)$ and $(2,4)$.

\begin{tabular}{|c|c|c|}
\hline Graph & Connected components & Relators \\
\hline \hline$G_{(3,2)}$ & $\{(2,0),(1,2)\},\{(0,1),(3,1)\}$ & $e_{1}+e_{3}=e_{2}+e_{4}$ \\
\hline$G_{(6,2)}$ & $\{(2,0),(0,1)\},\{(3,1)\}$ & $3 e_{1}+2 e_{2}=2 e_{4}$ \\
\hline$G_{(4,3)}$ & $\{(2,0),(0,1)\},\{(1,2),(3,1)\}$ & $2 e_{1}+3 e_{2}=e_{3}+e_{4}$ \\
\hline$G_{(2,4)}$ & $\{(2,0),(0,1)\},\{(1,2)\}$ & $e_{1}+4 e_{2}=2 e_{3}$ \\
\hline
\end{tabular}

Hence

$$
\begin{aligned}
\rho= & (((1,0,1,0),(0,1,0,1)),((3,2,0,0),(0,0,0,2)), \\
& ((2,3,0,0),(0,0,1,1)),((1,4,0,0),(0,0,2,0))\}
\end{aligned}
$$

is a minimal presentation of $S$.

In [10] the authors show that if $S$ is a Cohen-Macaulay simplicial affine semigroup with minimal Apéry set (there called with maximal codimension), then $\# \rho=m(m+1) / 2$. Moreover, this property characterizes CohenMacaulay simplicial affine semigroup with minimal Apéry set. Let us see what happens in the Buchsbaum case.

Theorem 13. Let $S$ be a Buchsbaum simplicial affine semigroup with minimal Apéry set. Let $\sim$ be the equivalence relation defined over $\bigcap_{i=1}^{r} \mathrm{~S}\left(n_{i}\right)$ as before and $\lambda=\#\left\{[x] \in \bigcap_{i=1}^{r} \mathrm{~S}\left(n_{i}\right) / \sim: \#[x]=r\right\}$. For every minimal system of presentation $\rho$ of $S$,

$$
\# \rho=\frac{m(m+1)}{2}+\lambda \frac{r(r-1)}{2} .
$$


Proof. As we have indicated before, every minimal system of generators has the same cardinality. Hence it suffices to count the elements belonging to $\rho=\bigcup_{n \in S} \rho_{n}$. For doing this, we must know which are the elements in $S$ fulfilling that $G_{n}$ is not connected. If $n \in S$ and $G_{n}$ is not connected, then this graph must contain a connected component with some of its vertices lying in $\left\{n_{1}, \ldots, n_{r}\right\}$, otherwise $n-n_{i} \notin S$ for all $i \in\{1, \ldots, r\}$ and thus $n \in \bigcap_{i=1}^{r} \mathrm{~S}\left(n_{i}\right)=\left\{0, n_{r+1}, \ldots, n_{r+m}\right\}$, contradicting that $\left\{n_{1}, \ldots, n_{r+m}\right\}$ is a minimal system of generators of $S$. For the rest of the proof and for a given $n \in S$ such that $G_{n}$ is not connected, we fix $G_{n}^{1}$ (defined in the description of $\rho$ given above) as one of these connected components of $G_{n}$ fulfilling that some of its vertices are contained in $\left\{n_{1}, \ldots, n_{r}\right\}$. From the construction of $\rho$, for every $n \in S$ and every component of $G_{n}$ other than $G_{n}^{1}$, we get a new element in $\rho$. It follows that in order to count the cardinality of $\rho$, we only have to decide how many connected components different from the fixed $G_{n}^{1}$ 's are in all the possible non-connected graphs $G_{n}$ 's. We first count those connected components in all the possible non-connected graphs not having vertices in $\left\{n_{1}, \ldots, n_{r}\right\}$ (these are of course different from any $G_{n}^{1}$ ) and then we will count those connected components having some vertices in $\left\{n_{1}, \ldots, n_{r}\right\}$ and different from the fixed $G_{n}^{1}$ 's.

Take $n \in S$ such that $G_{n}$ is not connected and contains a connected component $C$ whose vertices belong to $\left\{n_{r+1}, \ldots, n_{r+m}\right\}$. Then $n$ can be expressed as $n=\sum_{i=1}^{m} a_{i} n_{r+i}$ with $\left(a_{1}, \ldots, a_{m}\right) \in \mathbb{N}^{m}$. Observe that $\sum_{i=1}^{m} a_{i} \geq 2$, since otherwise $n \in \bigcap_{i=1}^{r} \mathrm{~S}\left(n_{i}\right)$. We claim that $\sum a_{i}=2$. If this were not the case, then there would exist $i, j, k \in\{1, \ldots, m\}$ (maybe not different) such that $n=n_{r+i}+n_{r+j}+n_{r+k}+s$, for some $s \in\left\langle n_{r+1}, \ldots, n_{r+m}\right\rangle$. Since $n_{r+i}+n_{r+j} \notin \bigcap_{i=1}^{r} \mathrm{~S}\left(n_{i}\right)$, there exists $l \in\{1, \ldots, r\}$ such that $n_{r+i}+$ $n_{r+j}-n_{l} \in S$. However, this leads to $n-\left(n_{r+k}+n_{l}\right) \in S$, which implies that $n_{l}$ is a vertex of $C$, contradicting $\mathrm{V}(C) \subseteq\left\{n_{r+1}, \ldots, n_{r+m}\right\}$. Hence $n$ must be of the form $n=n_{r+i}+n_{r+j}$ with $i, j \in\{1, \ldots, m\}$. Conversely, since $n_{r+i}+n_{r+j} \notin \bigcap_{i=1}^{r} \mathrm{~S}\left(n_{i}\right)$ for all $i, j \in\{1, \ldots, m\}$, each element of this form yields an element in $\rho$. In this way we collect $m(m+1) / 2$ elements in $\rho$.

Now we determine for which $n \in S$ the graph $G_{n}$ has at least two connected components containing vertices belonging to $\left\{n_{1}, \ldots, n_{r}\right\}$ (recall that one of these was taken to be $G_{n}^{1}$ ). If $n$ fulfills this condition, then there must exist $i, j \in\{1, \ldots, r\}$ such that $n-n_{i}, n-n_{j} \in S$ and $n-\left(n_{i}+n_{j}\right) \notin S\left(n_{i}\right.$ and $n_{j}$ are in different connected components of $\left.G_{n}\right)$. Since $S$ is Buchsbaum, Theorem 5 ensures that $\bar{S}$ is Cohen-Macaulay. The elements $n-n_{i}, n-n_{j}$ belong to $S$, which implies that they belong to $\bar{S}$ and by Proposition 8 , we obtain that $n-\left(n_{i}+n_{j}\right) \in \bar{S}$. This leads to $n-\left(n_{i}+n_{j}\right)=m \in \bar{S} \backslash S$. As we did in the proof of Theorem 9 , it is easy to show that $\left\{m+n_{1}, \ldots, m+n_{r}\right\} \subseteq \bigcap_{i=1}^{r} \mathrm{~S}\left(n_{i}\right)=\left\{0, n_{r+1}, \ldots, n_{r+m}\right\}$. Since $n-n_{i}=m+n_{j}$ and $n-n_{j}=m+n_{i}$, there exists $s, t \in\{1, \ldots, m\}$ such that 
$n=n_{r+t}+n_{j}=n_{r+s}+n_{i}=\left(m+n_{i}\right)+n_{j}=\left(m+n_{j}\right)+n_{i}$, which implies that $n_{r+s} \in\left[n_{r+t}\right]$ and thus $\#\left[n_{r+t}\right]=r=\#\left[n-n_{i}\right]$. In addition, $m-n_{k} \notin \bar{S}$ for all $k \in\{1, \ldots, r\}$, since otherwise $\left(m-n_{k}\right)+n_{k}=m$ should belong to $S$. Hence $m \in \bigcap_{i=1}^{r} \overline{\mathrm{S}}\left(n_{i}\right)$. By Proposition 8 , if there exists $m^{\prime} \in \bar{S} \backslash S$ and $i^{\prime}, j^{\prime} \in\{1, \ldots, r\}$ such that $n=\left(m+n_{i}\right)+n_{j}=\left(m^{\prime}+n_{i}^{\prime}\right)+n_{j}^{\prime}$, then $m$ must be equal to $m^{\prime}$ and $\{i, i\}=\left\{i^{\prime}, j^{\prime}\right\}$. This implies that in this case there are exactly two connected components of $G_{n}$ with some of its vertices in $\left\{n_{1}, \ldots, n_{r}\right\}$ (and this yields a new element in $\rho$ ). Thus for a fixed $m$, we get as many new elements in $\rho$ as elements of the form $\left(m+n_{i}\right)+n_{j}$ we can write with $i<j$. This makes $r(r-1) / 2$ new elements in $\rho$. Moreover, for each element $x \in \bigcap_{i=1}^{r} \mathrm{~S}\left(n_{i}\right)$ such that $\#[x]=r$, we get an element $m$ as before. It follows that we obtain $\lambda r(r-1) / 2$ elements in $\rho$ corresponding to the graphs having at least two connected components (and therefore exactly two) with some of its vertices lying in $\left\{n_{1}, \ldots, n_{r}\right\}$.

We conclude that $\# \rho=m(m+1) / 2+\lambda r(r-1) / 2$.

\section{References}

[1] H. Bresinsky, Monomial ideals in $\mathbb{P}^{3}$, Manuscripta Math., 47 (1984), 105-132, MR 85j:14090, Zbl 0547.13014.

[2] H. Bresinsky, P. Schenzel and W. Vogel, On liaison, arithmetical Buchsbaum curves and monomial curves in $\mathbb{P}^{3}$, J. of Algebra, 86 (1984), 283-301, MR 85c:14031, Zbl 0532.14016.

[3] P.A. García-Sánchez, Semigrupos Afines, Tesis Doctoral, Universidad de Granada, 1996.

[4] S. Goto, Buchsbaum rings of maximal embedding dimension, J. of Algebra, 76 (1982), 383-399, MR 83k:13014, Zbl 0482.13012.

[5] S. Goto, N. Suzuki and K. Watanabe, On affine semigroup rings, Japan J. Math., 2 (1976), 1-12, MR 56 \#8553, Zbl 0361.20066.

[6] J. Herzog, Generators and relations of Abelian semigroups and semigroup rings, Manuscripta Math., 3 (1970), 175-193, MR 42 \#4657, Zbl 0211.33801.

[7] L.T. Hoa, Algorithmical aspects of the problems of classifying multi-projections of Veronesean varieties, Manuscripta Math., 63 (1989), 317-331.

[8] Y. Kamoi, Defining ideals of Buchsbaum semigroup rings, Nagoya Math. J., 136 (1994), 115-131, MR 96a:13028, Zbl 0810.13026.

[9] J.C. Rosales, An algorithmic method to compute a minimal relation for any numerical semigroup, Internat. J. Algebra Comput., 6 (1996), 441-455, MR 97f:20080, Zbl 0863.20026.

[10] J.C. Rosales and P.A. García-Sánchez, On Cohen-Macaulay and Gorenstein simplicial affine semigroups, Proc. Edinburgh Math. Soc., 41 (1998), 517-537, MR 2000e:20107, Zbl 0904.20048.

[11] J.C. Rosales, P. A. García-Sánchez and J. M. Urbano-Blanco, On presentations of commutative monoids, Internat. J. Algebra Comput., 9 (1999), 539-553, MR 2000h:20102. 
[12] P. Schenzel, On Veronesean embeddings and projections of Veronesean varieties, Arch. Math., 30 (1978), 391-397, MR 58 \#5651, Zbl 0417.14040.

[13] J. Stückrad and W. Vogel, Buchsbaum Rings and Applications, Springer-Verlag, 1986, MR 88h:13011b, Zbl 0606.13018.

[14] N.V. Trung, Classification of the double projections of Veronese varieties, J. Math. Kyoto Univ., 22 (1983), 567-581, Zbl 0511.14024.

[15] _ Projections of one-dimensional Veronese varieties, Math. Nechr., 118 (1984), 47-67, Zbl 0584.14034.

[16] N.V. Trung and L.T. Hoa, Affine semigroups and Cohen-Macaulay rings generated by monomials, Trans. Amer. Math. Soc., 298 (1986), 145-167, Zbl 0631.13020.

Received April 24, 2000 and revised September 6, 2000. This paper was supported by the project DGES PB96-1424.

Departamento de Álgebra

UNIVERSIDAD DE GRANADA

E-18071 Granada, Spain

E-mail address: pedro@ugr.es

Departamento de Álgebra

UNIVERSIDAD DE GRANADA

E-18071 Granada, Spain

E-mail address: jrosales@ugr.es 